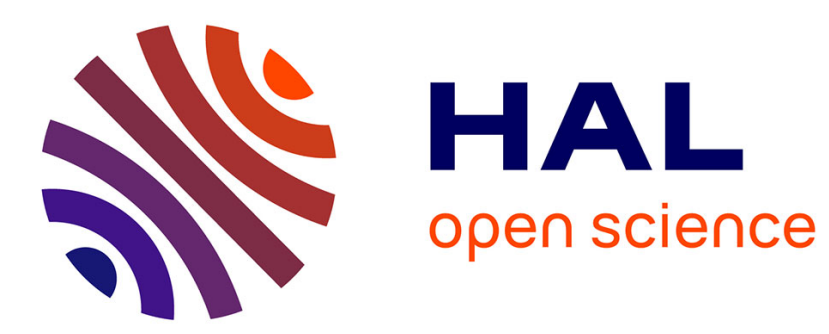

\title{
CCD astrometric observations of Amalthea and Thebe in the Gaia era
}

\author{
Vincent Robert, E. Saquet, J.-E Arlot, François Colas
}

\section{To cite this version:}

Vincent Robert, E. Saquet, J.-E Arlot, François Colas. CCD astrometric observations of Amalthea and Thebe in the Gaia era. Monthly Notices of the Royal Astronomical Society, 2017, 10.1093/mn$\mathrm{ras} / \mathrm{stx} 123$. hal-01458954

\section{HAL Id: hal-01458954 https://hal.sorbonne-universite.fr/hal-01458954}

Submitted on 7 Feb 2017

HAL is a multi-disciplinary open access archive for the deposit and dissemination of scientific research documents, whether they are published or not. The documents may come from teaching and research institutions in France or abroad, or from public or private research centers.
L'archive ouverte pluridisciplinaire HAL, est destinée au dépôt et à la diffusion de documents scientifiques de niveau recherche, publiés ou non, émanant des établissements d'enseignement et de recherche français ou étrangers, des laboratoires publics ou privés. 


\title{
CCD astrometric observations of Amalthea and Thebe in the Gaia era ${ }^{\star}$
}

\author{
V. Robert ${ }^{1,2} \dagger$, E. Saquet ${ }^{1,2}$, F. Colas $^{2}$, J.-E. Arlot ${ }^{2}$ \\ ${ }^{1}$ Institut Polytechnique des Sciences Avancées IPSA, 63 boulevard de Brandebourg, F-94200 Ivry-sur-Seine, France \\ ${ }^{2}$ Institut de Mécanique Céleste et de Calcul des Éphémérides IMCCE, Observatoire de Paris, PSL Research University, CNRS, Sorbonne \\ Universités, UPMC Univ. Paris 06, Univ. Lille 1, 77 av. Denfert-Rochereau F-75014 Paris, France
}

Accepted XXX. Received YYY; in original form ZZZ

\begin{abstract}
In the framework of the 2014-2015 campaign of mutual events, we observed Jupiter's inner satellites Amalthea (JV) and Thebe (JXIV). We focused on estimating whether the positioning accuracy determined from direct astrometry could compete with that derived from photometric observations of eclipses, for dynamical purposes.

We present the analysis of 35 observations of Amalthea and 19 observations of Thebe realized with the 1-m telescope at Pic du Midi observatory during three nights in 2015, January and April. The images were reduced through an optimal process that includes image and spherical corrections using the Gaia-DR1 catalog to provide the most accurate equatorial (RA, Dec) positions. We compared the observed positions of both satellites with the theoretical positions from JPL JUP310 satellite ephemerides and from IMCCE INPOP13c planetary ephemeris. The rms (O-C) in equatorial positions are \pm 112 mas for the Amalthea observations, or $330 \mathrm{~km}$ at Jupiter, and \pm 90 mas for the Thebe observations, or $270 \mathrm{~km}$ at Jupiter. Using the Gaia-DR1 catalog allowed us to eliminate systematic errors due to the star references up to 120 mas, or $350 \mathrm{~km}$ at Jupiter, by comparison with the UCAC4 catalog.
\end{abstract}

Key words: astrometry - ephemerides - planets and satellites: individual: Amalthea - planets and satellites: individual: Thebe

\section{INTRODUCTION}

Amalthea (JV) and Thebe (JXIV) are the two most massive of the four inner Jovian moons. The biggest and brightest satellite Amalthea was first discovered from the ground by Barnard (1892) with the 36-inch refractor at Lick Observatory, USA. It orbits around the planet at a distance of 2.54 Jupiter radii $R_{J}$. Its visual magnitude is 14.1 . Thebe and the two other inner moons Metis and Adrastea, were discovered nearly one century after (Jewitt et al. 1979; Stone \& Synnott 1980; Synnott 1980, 1981), when the Voyager 1 and 2 spacecraft observed the inner system of Jupiter. Thebe orbits around the planet at a distance of $3.11 R_{J}$. Its visual magnitude is 15.7 .

Since Amalthea and Thebe are faint and close to Jupiter, they are difficult targets for ground-based observations. The brightness of the planet introduces a high background noise that needs particular observational techniques

^ Based on observations made at Pic du Midi observatory (S2P), France. Full Tables 2 and 3 are available in electronic form at the Natural Satellites DataBase and Natural Satellites Data Center services of IMCCE via http://nsdb.imcce.fr/ or http://www.imcce.fr/nsdc/

$\dagger$ E-mail: vincent.robert@obspm.fr to make the satellites visible and measurable (Colas 1991). This explains first the lack of data available for these bodies, and as a consequence, the lack of precision in their respective dynamical models.

The first observers determined orbital parameters of Amalthea (Barnard 1892) and Thebe (Synnott 1984), while adjusting simple models to very few observations. Since the fifth satellite of Jupiter was discovered long before, it has been more observed and studied than Thebe. However, the data available for this last one were accurate enough to determine its orbit as precisely as for Amalthea. Later, the first reliable dynamical models were produced by Jacobson (1994) using the formulas of precessing orbits, while the Galileo spacecraft observed the four inner moons and revealed, in particular, that Amalthea and Thebe determined the structure of the gossamer rings (Burns et al. 1999). Afterward, improvements consisted of successive adjustments to the theories while the sets of data were completed with CCD (Kulyk et al. 2002; Veiga \& Vieira Martins 2005), Cassini spacecraft (Cooper et al. 2006) and HST observations (Mallama et al. 2004), but the difficulties of observing such faint bodies and representing their motion remained (Avdyushev \& Ban'shikova 2008). It is obvious that new observations will help in improving our 
Table 1. Raw statistics of the Pic du Midi observatory observations of Amalthea and Thebe. Column A denotes the number of observations of Amalthea, $\mathrm{T}$ the number of observations of Thebe, and G the number of Gaia-DR1 reference stars used for the astrometric calibration.

\begin{tabular}{ccccc}
\hline \hline Night & Exp. time (s) & A & T & G \\
\hline $2015-01-05$ & $30-60$ & 10 & - & 4 \\
$2015-01-06$ & $30-60$ & - & 19 & 4 \\
$2015-04-06$ & $30-60$ & 25 & - & 4 \\
\hline
\end{tabular}

knowledge of both the satellite dynamics and the ring system. However, the last available astrometric data were published by Kulyk (2008), and the last photometric data by Christou et al. (2010).

During the 2014-2015 campaign of mutual events, we recorded ground-based photometric observations of eclipses of Amalthea and, for the first time, Thebe by the Galilean moons (Saquet et al. 2016). The success of this work and the value of the positioning results motivated us to compare the photometric accuracy with that derived from direct astrometry of new ground-based observations, using the most recent Gaia-DR1 (Lindegren et al. 2016) star catalog and astrometric reduction techniques (Robert 2011).

\section{OBSERVATIONS AND REDUCTIONS}

35 observations of Amalthea and 19 observations of Thebe were realized at Pic du Midi observatory during three nights in 2015, January and April. The exposure times of the images are $30-60 \mathrm{~s}$. The field of view is $5.45 \mathrm{arcmin}$ on both the $x$-axis and the $y$-axis. Table 1 summarizes the exposure data for the series.

Fig. 1 shows a typical CCD observation. This 30 -second exposure of Jupiter was taken with the 1-m telescope during our first night of observation. An Andor Ikon-L camera, in combination with a Schott RG695 lowpass filter was used to limit the diffusion light at short wavelengths, and to get a lower contrast with Jupiter (Colas 1991). A small mask on the filter covers the planet, avoiding large blooming artefacts. Extra illumination is clearly seen in the Jupiter area because we did not use a real coronagraph but a suitable dot deposited on the filter. In consequence, additional light remains on the image, due to the diffusion in the different optical parts of the telescope. The satellites visible in this exposure are, from left to right, Europa and Io (over-exposed), and Amalthea. Thebe is close to Jupiter, and behind the mask. North is up, East to the left.

Before extracting the inner satellite and star positions, we removed the background noise in each area of the object to be measured. We isolated the objects in small zones and fitted individual sky backgrounds with three-degree to five-degree surface polynomials by least-squares, in order to subtract them from the images. The polynomial degrees were dependent on the light gradient in the zones. Fig. 2 shows the sky background in the Amalthea zone of Fig. 1 (left), and the corresponding subtraction result (right). We can see that we were able to produce a visible and measurable image of the satellite.

Measurement and reduction techniques were the

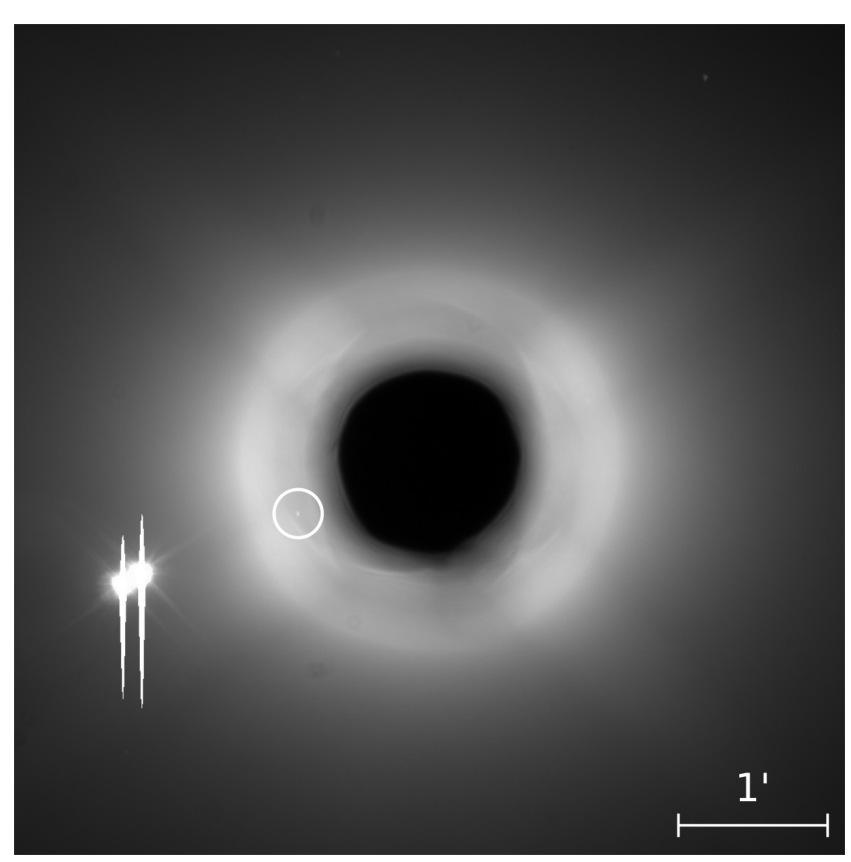

Figure 1. Typical CCD observation of Amalthea at Pic du Midi observatory. From left to right: Europa and Io (over-exposed), and Amalthea (displayed in the white circle). The dark shape at the center is the shadow of the small mask. Thebe is close to Jupiter, and behind the mask. North is up, East to the left. The field of view is 5.45 arcmin on both the $x$-axis and the $y$-axis.
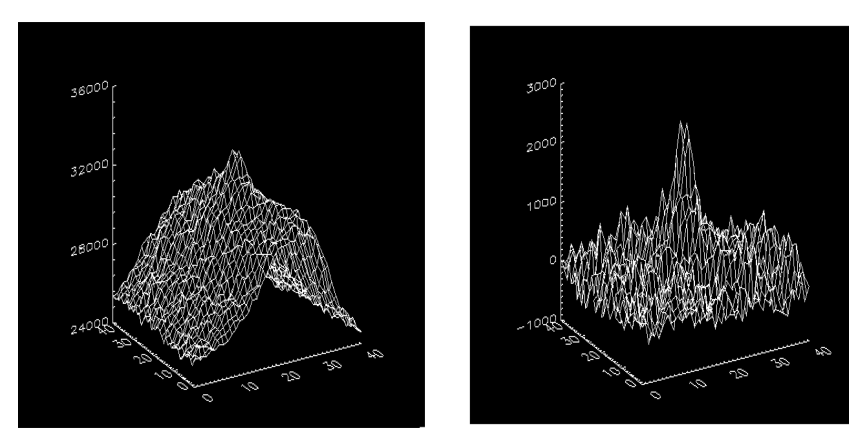

Figure 2. Sky background measure in the Amalthea zone of Fig. 1 (left). The horizontal $x$-axis and $y$-axis show the pixel position in the area, and the vertical $z$-axis shows the pixel value in ADU. The fitted surface polynomial was subtracted from the image to make the satellite visible and measurable while avoiding errors in the position extraction (right).

same as described in Robert et al. (2015). Measured $(x, y)$ image positions were corrected for spherical effects. The observations contain four Gaia-DR1 reference stars (Lindegren et al. 2016), and the reductions were performed using suitable four constant functional models to provide equatorial (RA, Dec) astrometric positions of the satellites. Scale $\rho$, orientation $\theta$, and offsets $\Delta x$ and $\Delta y$ were modeled for the determination of the tangential $(X, Y)$ coordinates. All our observations were equatorial (RA, Dec) astrometric positions obtained from tangential $(X, Y)$ coordinates by using the gnomonic inverse projection and determined in an ICRS geocentric reference frame to be easily compared with the most recent ephemerides. 

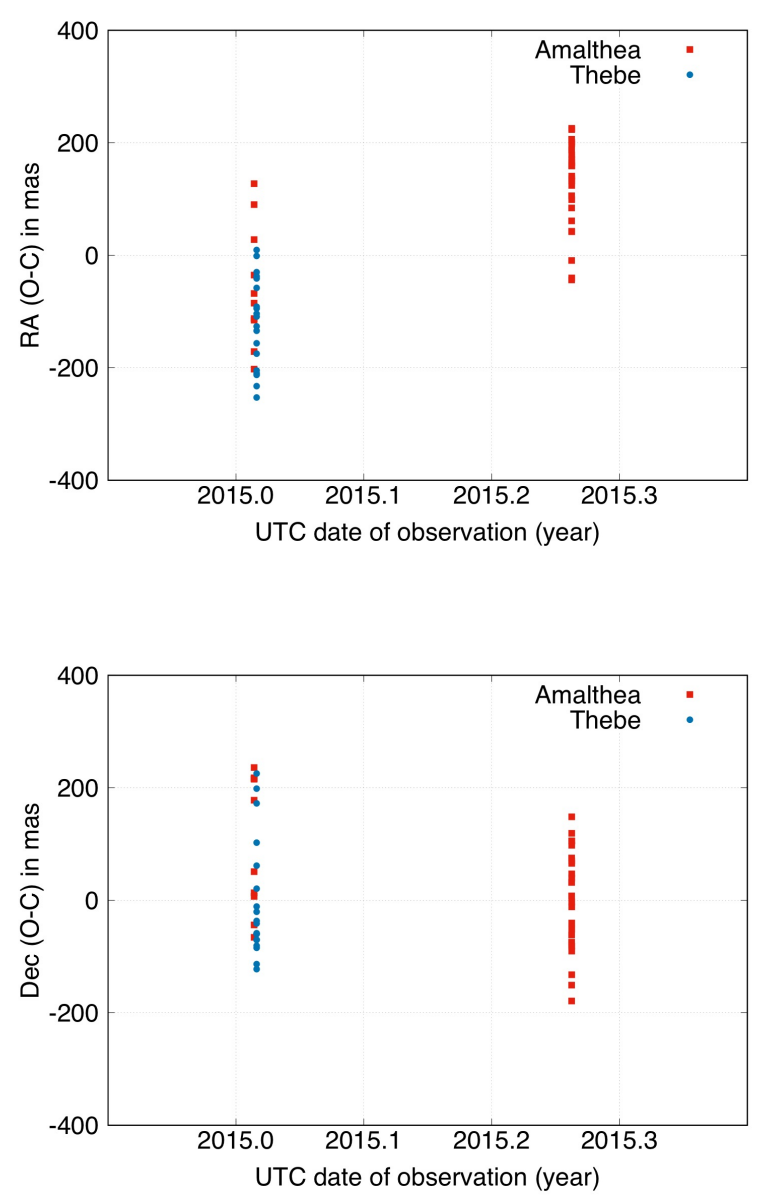

Figure 3. (RA, Dec) (O-C) according to JUP310 and INPOP13c ephemerides. The $x$-axis shows the UTC date of observation and $y$-axis the RA and Dec (O-C). Red squares denote Amalthea, and blue circles Thebe.

\section{POSITIONING RESULTS}

We compared the 35 positions of Amalthea and the 19 positions of Thebe with their theoretical computed positions given by the JPL JUP310 satellite ephemerides (Jacobson 2013) and the IMCCE INPOP13c planetary ephemeris (Fienga et al. 2014). The positioning results are provided in Tables 2 and 3 for both satellites, respectively. They are available in electronic form at the IMCCE as well. Starting from the lefthand column, we provide the mean TDB date of observation in Julian Days, the geocentric observed right ascension, and declination in degrees. The (O-C) distributions of residuals in equatorial right ascension and declination are provided in Figs. 3 and 4, and in Tables 4 and 5. They show the difference of (RA, Dec) coordinates for individual satellites, hence the observed positions versus positions calculated from INPOP13c ephemeris.

Offsets for each night, visible in Figs. 3 and 4, show large differences between the right ascension and declination (O-C), for each satellite. The mean RA (O-C) values for Amalthea are shifted by about -55 mas and 125 mas, for the first and second observation nights, respectively. Its mean Dec (O-C) value is shifted by about 100 mas for the

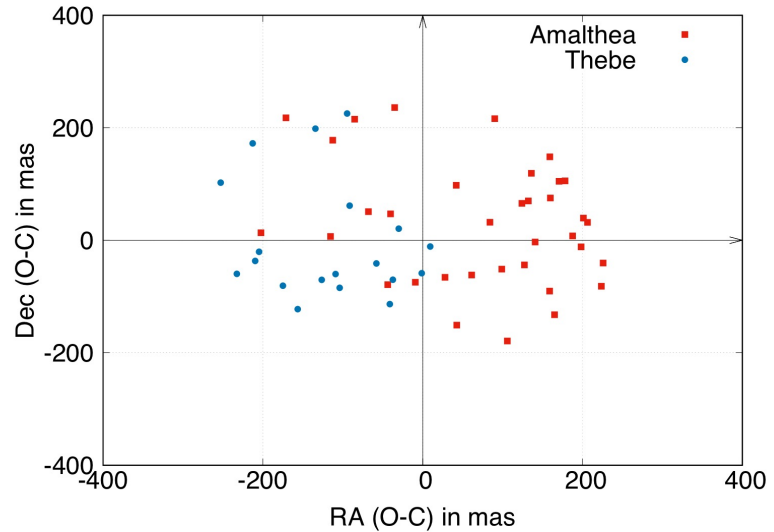

Figure 4. (RA, Dec) (O-C) according to JUP310 and INPOP13c ephemerides. The $x$-axis shows the RA (O-C) and $y$-axis the Dec (O-C). Red squares denote Amalthea, and blue circles Thebe.

Table 2. Astrometric positions of Amalthea (JV). The full table is available in electronic form at the IMCCE.

\begin{tabular}{ccc}
\hline \hline Date (TDB) & RA (deg.) & Dec (deg.) \\
\hline 2457028.696810 & 143.813188 & 15.279286 \\
2457028.697181 & 143.813120 & 15.279310 \\
2457028.697924 & 143.813164 & 15.279375 \\
2457028.698295 & 143.813138 & 15.279313 \\
2457028.698666 & 143.813148 & 15.279328 \\
2457028.699408 & 143.813187 & 15.279378 \\
2457028.699780 & 143.813193 & 15.279308 \\
2457028.700151 & 143.813128 & 15.279383 \\
2457028.702368 & 143.813071 & 15.279401 \\
2457028.704224 & 143.813052 & 15.279407 \\
2457119.332483 & 135.136185 & 18.002290 \\
2457119.333996 & 135.136303 & 18.002191 \\
2457119.335509 & 135.136456 & 18.002134 \\
2457119.338536 & 135.136814 & 18.001998 \\
2457119.339293 & 135.136872 & 18.001979 \\
2457119.340806 & 135.137068 & 18.001979 \\
2457119.341725 & 135.137121 & 18.001948 \\
2457119.342481 & 135.137186 & 18.001898 \\
2457119.344751 & 135.137431 & 18.001845 \\
2457119.345508 & 135.137496 & 18.001849 \\
2457119.346264 & 135.137498 & 18.001809 \\
2457119.347021 & 135.137624 & 18.001803 \\
2457119.347777 & 135.137685 & 18.001792 \\
2457119.348534 & 135.137741 & 18.001762 \\
2457119.349290 & 135.137775 & 18.001735 \\
2457119.350046 & 135.137889 & 18.001675 \\
2457119.350803 & 135.137921 & 18.001685 \\
2457119.351560 & 135.137987 & 18.001620 \\
2457119.352316 & 135.138056 & 18.001622 \\
2457119.353830 & 135.138167 & 18.001597 \\
2457119.354586 & 135.138210 & 18.001530 \\
2457119.356856 & 135.138352 & 18.001530 \\
2457119.357612 & 135.138393 & 18.001480 \\
2457119.362153 & 135.138697 & 18.001375 \\
2457119.363666 & 135.138752 & 18.001367 \\
\hline & &
\end{tabular}


Table 3. Astrometric positions of Thebe (JXIV). The full table is available in electronic form at the IMCCE.

\begin{tabular}{ccc}
\hline \hline Date (TDB) & RA (deg.) & Dec (deg.) \\
\hline 2457029.411664 & 143.719943 & 15.313867 \\
2457029.413352 & 143.719727 & 15.314000 \\
2457029.414095 & 143.719649 & 15.313964 \\
2457029.414466 & 143.719654 & 15.314057 \\
2457029.414837 & 143.719634 & 15.313977 \\
2457029.415208 & 143.719580 & 15.314006 \\
2457029.415579 & 143.719504 & 15.314065 \\
2457029.417321 & 143.719357 & 15.314095 \\
2457029.418063 & 143.719305 & 15.314093 \\
2457029.418434 & 143.719316 & 15.314124 \\
2457029.418806 & 143.719247 & 15.314134 \\
2457029.419177 & 143.719191 & 15.314156 \\
2457029.420290 & 143.719125 & 15.314181 \\
2457029.420661 & 143.719097 & 15.314235 \\
2457029.421032 & 143.719041 & 15.314208 \\
2457029.421403 & 143.719063 & 15.314240 \\
2457029.421774 & 143.719013 & 15.314244 \\
2457029.422146 & 143.718990 & 15.314273 \\
2457029.422517 & 143.718929 & 15.314335 \\
\hline
\end{tabular}

Table 4. Statistics of the (RA, Dec) (O-C) for Amalthea (JV), in mas, according to JUP310 and INPOP13c ephemerides.

\begin{tabular}{ccccc}
\hline \hline Date & $\overline{(\boldsymbol{O}-C)_{\alpha \cos \delta}}$ & $\begin{array}{c}\sigma_{\alpha \cos \delta} \\
+/-\end{array}$ & $\overline{(\boldsymbol{O - C})_{\delta}}$ & $\begin{array}{c}\sigma_{\delta} \\
+/-\end{array}$ \\
\hline $2015-01-05$ & -54.4 & 102.4 & 102.4 & 114.8 \\
2015-04-06 & 124.3 & 76.5 & -0.4 & 88.5 \\
Overall & 73.2 & 117.0 & 30.0 & 107.3 \\
\hline
\end{tabular}

Table 5. Statistics of the (RA, Dec) (O-C) for Thebe (JXIV), in mas, according to JUP310 and INPOP13c ephemerides.

\begin{tabular}{ccccc}
\hline \hline Date & $\overline{(\boldsymbol{O}-C)_{\alpha \cos \delta}}$ & $\begin{array}{c}\sigma_{\alpha \cos \delta} \\
+/-\end{array}$ & $\overline{(\boldsymbol{O}-C)_{\delta}}$ & $\begin{array}{c}\sigma_{\delta} \\
+/-\end{array}$ \\
\hline $2015-01-06$ & -119.0 & 78.3 & -2.6 & 102.6 \\
\hline
\end{tabular}

first observation night, only. Concerning Thebe, we report that only the mean RA (O-C) value is shifted by about 120 mas. We conclude that the exposure timing can be ruled out. The camera shutter was triggered with a GPS card to ensure a timing precision of $1 \mathrm{~ms}$. However, we calibrated the three night sets of images with three different sets of four faint stars. Moreover, they were not well-distributed around the center of field. We assume that the calibration process and result were accurate but not constrained enough (Robert 2011), and because we could not release a second confident scale factor to fit on both the $x$-axis and the $y$-axis, separately, we were not able to compensate the anisotropy due to the reference stars distribution. As a consequence, it is difficult to analyze such offsets for the (O-C) in both the equatorial coordinates. More observations will be necessary.

The key point is that the JUP310/INPOP13c rms (OC) for all observations of Amalthea is 112.1 mas, and 90.4 mas for Thebe. These average rms (O-C) correspond to our observation accuracies and could be compared to the most precise astrometric observations. Veiga \& Vieira Martins (2005) determined positioning results with about 280 observations for both satellites separately, over six nights, with rms errors of 144 mas for Amalthea, and 145 mas for Thebe. We thus improved the positioning accuracy by 30 mas for Amalthea, or $90 \mathrm{~km}$ at Jupiter, and 55 mas for Thebe, or $160 \mathrm{~km}$ at Jupiter. Later, Kulyk (2008) determined positioning results with about 50 observations with rms errors of 100 mas for Amalthea, and 150 mas for Thebe, however, only with intersatellite observations for which most of the systematic effects and those due to the planet position are eliminated.

Several factors contributed to the increase in precision. The observational technique and the small mask made the satellites visible and measurable, probably with little contribution to the precision in their positioning. The photographic treatment allowed us to remove the background noise, and to apply model centroids to the images. In addition, the astrometric reduction process with the accurate star catalog used made possible correcting for all spherical effects and obtaining precision in the equatorial positions.

During the 2014-2015 campaign of mutual events, we recorded ground-based photometric observations of eclipses of Amalthea and Thebe by the Galilean moons (Saquet et al. 2016). We provided astrometric results with an external precision of 20 mas for the eclipses of Amalthea, and 53 mas for that of Thebe. In spite of the improvements we made with the standard astrometric observations, the accuracies derived from photometric observations of eclipses largely override our measurements. This confirms that photometric records of mutual events still remain more precise than direct astrometry (Arlot et al. 2014).

\section{GAIA-DR1 VS UCAC4}

We finally compared our astrometric results with those determined using UCAC4 reference stars (Zacharias et al. 2013). Obviously, we used exactly the same sets of four stars for each night of observation, and thus the exact correspondances between the Gaia-DR1 and UCAC4 catalogs for the calibrations.

Table 6 shows the averaged (RA, Dec) (O-C) for Amalthea and Thebe using both the star catalogs. Mean rms (O-C) is 148 mas for UCAC4, and 122 mas for GaiaDR1. The mean difference in the positioning accuracy is up to $26 \mathrm{mas}$, or $80 \mathrm{~km}$ at Jupiter. The averaged (O-C) are even more significant to compare since they are affected by local systematic errors of individual reference stars. In particular, we can see that the mean Dec (O-C) is 137.3 mas for UCAC4, and 18.8 mas for Gaia-DR1. The mean difference for this coordinate is up to 120 mas, and highlights that the UCAC4 catalog could be affected by important local errors.

We confirm our last conclusion with Fig.5. We here investigate the robustness of the astrometric calibration using both the star catalogs with the scale factors, which are the main indicators that characterize the reduction model and its accuracy (Robert 2011; Robert et al. 2011). The individual observations from 1 to 10 are those of the first night on 05 January 2015. The individual observations from 11 to 29 are those of the second and successive night on 06 January 2015. For two successive nights, one would wait for the scale 
Table 6. Statistics of the averaged (RA, Dec) (O-C) of Amalthea and Thebe using the UCAC4 and Gaia-DR1 star catalogs, and according to JUP310 and INPOP13c ephemerides.

\begin{tabular}{ccccc}
\hline \hline Catalog & $\overline{(O-C)_{\alpha \cos \delta}}$ & $\begin{array}{c}\sigma_{\alpha \cos \delta} \\
+/-\end{array}$ & $\overline{(O-C)_{\delta}}$ & $\begin{array}{c}\sigma_{\delta} \\
+/-\end{array}$ \\
\hline UCAC4 & 7.4 & 103.4 & 137.3 & 193.3 \\
GAIA-DR1 & 9.9 & 138.2 & 18.8 & 105.3 \\
\hline
\end{tabular}

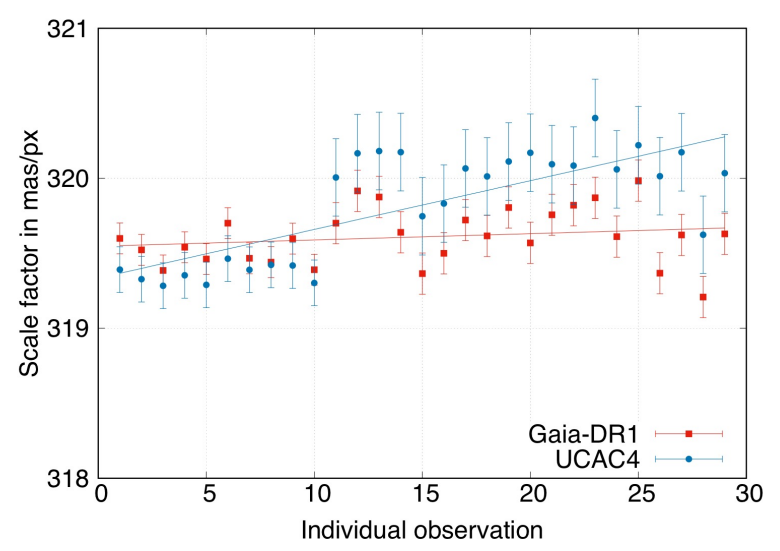

Figure 5. Scale factors derived from the astrometric calibrations of the observations on 05 and 06 January 2015. The $x$-axis shows the individual observation numbers and $y$-axis the fitted scale factor. Red squares denote calibrations with Gaia-DR1, and blue circles with UCAC4.

factors to be stable in evolution, even if the sets of reference stars used are quite different. This is the case for the calibrations with Gaia-DR1, but not for those with UCAC4. The evolution of the Gaia-DR1 scale factors shows a quite good stability, and we may deduce that the calibration is confident and accurate. The evolution of the UCAC4 scale factors shows an important bias between the two sets of both nights of observation, which is typical of local systematic errors. This effect could explain the bias in the mean UCAC4 Dec (O-C) visible in Table 6, as well. Moreover, the error bars are representative of the reference star formal errors. Because the UCAC4 errors on the scale factors are three times higher than the Gaia-DR1's, we may deduce that we eliminated the systematic and minimized the random errors due to the reference stars. Observational and measurement errors "only" remain at this point, and we confirm that astrometric calibration will be more confident with Gaia-DR1 in the future.

\section{CONCLUSION}

We analyzed three series of CCD observations of Amalthea and Thebe realized at Pic du Midi observatory in 2015, January and April. In spite of the difficulty to observe the inner satellites of Jupiter from the ground, we provided astrometric equatorial positions of the satellites better in accuracy than previous works with direct astrometry. The mean rms
(O-C) are 112 mas for Amalthea, and 90 mas for Thebe. Using the Gaia-DR1 catalog allowed us to improve the positional accuracy by 26 mas by comparison with the UCAC4 catalog, but most important, it allowed us to eliminate systematic errors due to the star references up to $\mathbf{1 2 0}$ mas. Even though we improved the standard astrometric accuracy, we confirm that photometric records of mutual events still remain more precise by a factor ranging from 2 to 5 . However, improvements in observational techniques and reduction processes using Gaia-DR1, the most accurate star catalog available, make direct astrometry an effective way to fill in the lack of data over continuous time spans, since mutual events can only occur every six years.

We plan to make new direct astrometry of these inner satellites to obtain more data and confirm our conclusions. We plan to re-analyze old data in the framework of the NAROO project ${ }^{1}$ as well. A new international center of digitization and analysis of old observations will be set up in Paris at the beginning of 2017. It is intended to digitize old astrophotographic plates with high accuracy, reanalyze the observations, produce new (RA, Dec) astrometric positions of the objects, and provide a free database for the community. Our main goal is to provide accurate positions of the planets, satellites, asteroids and comets over one century, with the best accuracy.

\section{REFERENCES}

Arlot J.-E., et al., 2014, A\&A, 572, A120

Avdyushev V. A., Ban'shikova M. A., 2008, Solar System Research, 42, 296

Barnard E. E., 1892, AJ, 12, 81

Burns J. A., Showalter M. R., Hamilton D. P., Nicholson P. D., de Pater I., Ockert-Bell M. E., Thomas P. C., 1999, Science, 284,1146

Christou A. A., Lewis F., Roche P., Hidas M. G., Brown T. M., 2010, A\&A, 522, A6

Colas F., 1991, PhD thesis, Observatoire de Paris,

Cooper N. J., Murray C. D., Porco C. C., Spitale J. N., 2006, Icarus, 181, 223

Fienga A., Manche H., Laskar J., Gastineau M., Verna A., 2014, IMCCE, Observatoire de Paris,

Jacobson R. A., 1994, JPL IOM, 314

Jacobson R. A., 2013, NASA NAIF Generic Kernels,

Jewitt D. C., Danielson G. E., Synnott S. P., 1979, Science, 206, 951

Kulyk I., 2008, Planet. Space Sci., 56, 1804

Kulyk I., Jockers K., Karpov N., Sergeev A., 2002, A\&A, 383, 724

Lindegren L., et al., 2016, preprint, (arXiv:1609.04303)

Mallama A., Aelion H. M., Mallama C. A., 2004, Icarus, 167, 320

Robert V., 2011, PhD thesis, Observatoire de Paris,

Robert V., et al., 2011, MNRAS, 415, 701

Robert V., Lainey V., Pascu D., Pasewaldt A., Arlot J.-E., De Cuyper J.-P., Dehant V., Thuillot W., 2015, A\&A, 582, A36

Saquet E., Emelyanov N., Colas F., Arlot J.-E., Robert V., Christophe B., Dechambre O., 2016, A\&A, 591, A42

Stone E. C., Synnott S. P., 1980, IAU Circ., 3470

Synnott S. P., 1980, Science, 210, 786

Synnott S. P., 1981, Science, 212, 1392

1 See the NAROO webpage of IMCCE and Paris Observatory at http://nsdb.imcce.fr/naroo/ 
Synnott S. P., 1984, Icarus, 58, 178

Veiga C. H., Vieira Martins R., 2005, A\&A, 437, 1147

Zacharias N., Finch C. T., Girard T. M., Henden A., Bartlett

J. L., Monet D. G., Zacharias M. I., 2013, AJ, 145, 44 